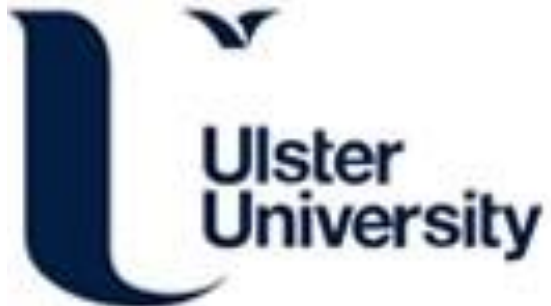

Multi-electrode systems for electrical impedance imaging

McAdams, ET., McLaughlin, JAD., \& Anderson, JMCC. (1999). Multi-electrode systems for electrical impedance imaging. Physiological Measurement, 15(2A), A101. https://doi.org/10.1088/0967-3334/15/2A/014

Link to publication record in Ulster University Research Portal

\section{Published in:}

Physiological Measurement

Publication Status:

Published (in print/issue): 01/01/1999

DOI:

10.1088/0967-3334/15/2A/014

\section{Document Version}

Publisher's PDF, also known as Version of record

\section{General rights}

Copyright for the publications made accessible via Ulster University's Research Portal is retained by the author(s) and / or other copyright owners and it is a condition of accessing these publications that users recognise and abide by the legal requirements associated with these rights.

\section{Take down policy}

The Research Portal is Ulster University's institutional repository that provides access to Ulster's research outputs. Every effort has been made to ensure that content in the Research Portal does not infringe any person's rights, or applicable UK laws. If you discover content in the Research Portal that you believe breaches copyright or violates any law, please contact pure-support@ulster.ac.uk. 
Multi-electrode systems for electrical impedance imaging

This article has been downloaded from IOPscience. Please scroll down to see the full text article.

1994 Physiol. Meas. 15 A101

(http://iopscience.iop.org/0967-3334/15/2A/014)

View the table of contents for this issue, or go to the journal homepage for more

Download details:

IP Address: 193.61.144.8

The article was downloaded on 24/05/2010 at 13:07

Please note that terms and conditions apply. 


\title{
Multi-electrode systems for electrical impedance tomography
}

\author{
E T McAdams, $\mathrm{J}$ A McLaughlin and $\mathrm{J} \mathrm{McC}$ Anderson \\ The Northern lreland Bio-Engineering Centre, University of Ulster, Shore Road, \\ Newtownabbey, Co Antrim BT37 OQB, UK
}

\begin{abstract}
A wide range of electrode system designs is reviewed for their possible use in aIT imaging.
\end{abstract}

\section{Introduction}

With the advances made in electrical impedance tomography (EIT), many research groups are now seeking to apply their EIT systems in vivo rather than on various physical phantoms.

In order to obtain an electrical impedance tomogram of a cross section through part of the body, impedance measurements must be made using an array of electrodes surrounding the part of the body under investigation, the number and relative position of the electrodes depending on the given EIT system. The accurate individual application of a large number of electrodes to a patient is tedious and time consuming and could prove a major drawback to the routine clinical use of EIT.

Of interest in EIT is an electrode system that

(i) adheres the electrodes to the body,

(ii) accurately locates the electrodes and

(iii) accommodates variations in the body segment's contours or dimensions due to, for example, respiration or patient movement.

An extensive survey of patients, journals, trade brochures etc was therefore undertaken in order to source potentially useful multi-electrode system designs. The most interesting tend to have been designed for ECG body surface mapping; some of these are presented below.

\section{Methods of electrode attachment}

In the past, adhesive-backed, rigid plastic ECG electrodes were adhered to the skin by means of a small annulus of double-sided adhesive tape placed under the outer flange of the plastic housing. The electrode sensor and the gel-impregnated sponge were located within the rigid housing in an effort to reduce artefacts due to mechanical disturbance of the electrode-electrolyte interface. These electrodes were generally reusable, with the geI and adhesive tape being replaced prior to reapplication.

In more recent low-cost, disposable ECG electrode designs, the rigid plastic housing has been removed and the sensor is now held in place on the patient by a surrounding disc of adhesive backing, expanded polythene or polyethylene foam. An important advantage in using foam is that it tends to absorb mechanical disturbance of the sensor, thus minimizing motion artefact. A possible disadvantage in using foam is that it does not permit the skin 
to breathe and can therefore give rise to skin irritation problems. Alternatives to expanded foam are microporous 'cloth' backings. These materials have minute pores that allow the skin to breathe and sweat. Cloth backings tend therefore to cause less skin irritation than foam and are often used for long-term monitoring applications. Cloth backings are also stretchable and, although this leads to patient comfort, the sensor can tend to pull away from the patient.

The recent use of adhesive hydrogel enables the design of low-cost, flexible electrodes. For many applications, the adhesive hydrogel dispenses with the need of a large surrounding disc of adhesive backing. This greatly simplifies the electrode design and the smaller electrodes can be placed closer together. As the gel is 'solid', there is less danger of electrical shorting between adjacent electrodes. Electrodes can be made with predetermined contact areas and geometries.

Mills and Stein (1981) patented an ECG electrode system, one embodiment of which involves placing an elastic chest piece, with embedded electrodes, on the chest of a reclining patient and attaching weights to either end of the chest piece to retain the electrodes in their correct anatomical positions and to ensure firm mechanical and electrical contact. We suggest that electrodes could also be placed under the subject, embedded in a compressible sheet, in order to completely encircle the body segment under investigation. In this latter case, body weight would ensure firm contact between the electrodes and the subject.

Hinsen et al (1979) have used a cardiac mapping system that consists of 48 dry metal electrodes mounted on a flexible substrate. The electrode system is applied to the patient's chest and, in order to ensure good electrical contact, weighted with a flexible lead plate. This is a further example of gravity being used to 'adhere' electrodes to a subject. A similar system, involving a flexible non-conductive silicone rubber sheet with embedded electrodes, is marketed for high-volume ECG recording by Polytechs Ltd, Tokyo, Japan.

Numerous researchers have used belts or vests of electrodes, which are fastened firmly around a body segment by means of, for example, various forms of buckle (Schwesinger $e t$ al 1984), snap coupling (Mills and Stein 1981) and Velcro pads (Stein 1983). The use of elasticated bands has also been reported (Toyosu 1983). In order to ensure optimal electrical contact with the patient when using such belts or bands, it is advantageous to use electrodes that protrude beyond the body of the belt (Mills and Stein 1981, Wen et al 1991). Yajima et al (1983), for example, constructed an electrode belt in which each electrode was mounted on a spring shaft.

Fischmann et al (1971) used a combination of spring-Ioaded and weight-activated electrodes mounted on a platform, which was lowered onto the chest of a lying patient. Arrays of spring-loaded electrodes have also been mounted onto frames against which the subject leans (Abraham 1983) or which are pressed against the subject (Knorre et al 1986). Kilpatrick et al (1979) describe a perspex cradle that fits around the thorax of a seated subject. On the cradle are radially mounted spring-loaded electrodes, which enable contact with the skin regardless of the shape of the subject's torso. Yajima et al (1983) used a 'cage' of spring-loaded electrodes, which closes around the thorax of a seated patient.

An interesting design has been patented by Romberg (1988). Electrodes are embedded in a multilayered vest, which has vertical hose-like air chambers that press the springloaded electrodes against the body upon inflation of the chambers. It is claimed that contact between the electrodes and the skin is made regardless of the body segment's size or shape.

Suction has also been used to adhere electrodes to the skin. One such electrode, commonly used in electrocardiography as the precordial lead, is the Welch bulb or suctioncup electrode (Welch 1951). It consists of a hollow, metallic, cylindrical electrode that makes contact with the skin at its base. A rubber suction bulb fits over the other end of the 
cylinder. In order to apply the electrode to the patient, the bulb is squeezed, the electrode is placed against the skin and the bulb is released, thus generating suction in the centre of the cylindrical electrode and holding the electrode assembly in place. Such electrodes tend to be used for short-term applications on subjects in a supine position. The relatively heavy structure tends to fall off subjects standing or sitting in an upright position. Since then, more suitable, light-weight, low-profile suction electrodes have been developed, which are pneumatically connected to remote bulbs (e.g. the Hillier chest electrode, now no longer manufactured) or vacuum pumps (Geddes et al 1976). Complete vacuum-suction multielectrode systems are now commercially available. For example, Vacumat VC1 is marketed by Medizinelektronik Thome of Gelnhausen, Germany for ECG monitoring.

Krenzke et al (1985) have patented an electrode system with electrodes connected to pneumatic pistons. The pistons are mounted radially on a horizontal cylindrical frame, which surrounds the patient. The couch on which the patient lies has apertures to enable electrode contact with the patient's back.

\section{Methods of rapidly and accurately locating electrodes}

Applying standard electrodes individually to a patient in the required positions is tedious and time consuming. To overcome this problem, several electrodes may be mounted, in their correct relative positions, onto a sheet of adhesive backing. For example, four conductive carbon electrodes are mounted on an adhesive foam backing and marketed by Survival Technology Inc. for long-term infant monitoring (InfanTrode).

In the past, a range of electrode systems has been designed involving belts, vests or sheets of backing material (e.g. canvas) into which were incorporated electrode leads and either (i) reusable electrodes (Hinsen et al 1979) or (ii) connectors into which electrodes could be inserted (Wen et al 1991). Standard belts/harnesses with integrated electrodes require the gelling of the electrodes prior to system application and the cleaning of the electrodes and the reapplication of double-sided adhesive annuli, following its removal (McAdams et al 1993). In the other form of beltharness, electrodes must be inserted into the provided connectors prior to application. Although these designs solve the problem of electrode location, application time and avoid lead entanglement, they require a considerable amount of time prior to and/or following system application. The problems of gelling and cleaning of electrodes mentioned above can obviously be avoided by using dry electrode systems. As electrode gel serves to moisten the skin site and ensure good electrical contact between the sensor and the patient, dry electrodes tend to give rise to large skin impedances with resultant electrical noise, interference and distortion problems. These problems can be greatly minimized by using an amplifier with a very high input impedance. However, the lead from the amplifier to the electrode, which has to be screened to avoid electrical interference, has a finite capacitance to earth and the attenuation thus caused limits the common-mode rejection ratio of the monitoring system (Betts and Brown 1976). By mounting buffer amplifiers directly on the back of the electrodes, this last problem can be avoided (Bergey et al 1971). Hinsen et al (1979), for example, have mounted 48 dry electrodes and their respective amplifiers in correct positions on a flexible, synthetic sheet. The electrode harness was applied to a subject and weighted with a flexible lead plate to ensure good contact between the electrodes and the patient's skin.

Kolesova et al (1982) and Abraham (1983) used suction electrode grids connected to vacuum pumps to adhere large numbers of electrodes for precordial ECG monitoring. The electrodes in Abraham's system were interconnected by fixed-length sections of soft 
'Silastic' tubing which held the electrodes in their correct relative positions and facilitated the application of large numbers (between 19 and 48) of electrodes to the chests of reclining subjects. A potential problem with vacuum-pump systems is that all electrodes must be applied simultaneously and their firm contact with the patient's skin ensured (Kolesova et al 1982). If one electrode is dislodged, all the electrodes are liable to fall off the subject. The application problem can be overcome, to some extent, by incorporating a device capable of disconnecting electrodes from the vacuum source when they were not applied to the subject. This, however, complicates the design and manufacture of the electrodes, increases their weight and makes them cumbersome (Kolesova et al 1982). Abraham (1983) minimized this problem by dividing his electrode matrix into pneumatically isolated sections. Suction was applied sequentially to each section, thus facilitating application. An alternative solution to this problem was suggested by Sippensgroenewegen et al (1986). Their suction electrode system is based on the venturi principle. Pressurized air is applied to a venturi ejector on each electrode and generates an underpressure within the electrode chamber, which compresses the electrode sensor against the patient's skin. A major advantage of this system over vacuum suction is that the dislodgement of one electrode will not disturb the suction of other electrodes.

Sippensgroenewegen et al (1986) applied seven arrays of venturi suction electrodes 'vertically' to the human thorax. The electrode arrays were each composed of a flat thin strap-like piece of flexible silicon rubber on which six or seven suction chambers, of the same material, were mounted. A total of 48 electrodes was applied. Although the 'vertical' interelectrode distances on each strap were fixed, the 'horizontal' distances between electrode arrays varied from patient to patient.

The spring-loaded electrode system of Kilpatrick et al (1979) involved a perspex 'cradle' which fitted around the thorax of a seated subject. On the cradle were mounted three rows of eight electrodes, which were free to move radially. The vertical separation of the three rows was adjustable while the horizontal distances between electrodes obviously depended on the size and shape of the thorax monitored.

The system of Krenzke et al (1985) comprises electrodes mounted onto pneumatic pistons which can converge radially on the subject's thorax. Once again, the 'horizontal' inter-electrode distances will depend on the size and shape of the body segment under study. An advantage, however, of this and similar designs is that, given the direction and extension of the electrode pistons, the positions of the electrodes can be computed. Inter-electrode distances and the shape of the body segment may then be derived from the above data and used in conjunction with the EIT algorithm to increase the accuracy of the EIT image.

\section{Methods of enabling inter-electrode movement}

During long-term EIT monitoring applications, the electrode system may be required to accommodate temporal changes in inter-electrode spacing and body segment shape and size due to, for example, respiration or subject movement. For many electrode system designs, the requirement that they can accommodate fluctuations in inter-electrode distances while on the patient is at odds with the requirement that the electrodes are initially held in their correct locations to ensure rapid, accurate application to the patient. Some compromises must therefore be made and these will depend on the application and the relative importance of the above requirements.

Inelastic harnesses can accommodate 'lateral' movement if several electrode bands or belts are placed in parallel on a given subject. This approach has been used by, for example, 
Yajima et al (1983) with spring-loaded electrodes mounted on belts; Sippensgroenewegen et al (1986) with venturi electrodes formed in flexible silicon rubber straps and Dempsey et al (1992) with strips of substrate on which sensors and leads had been screenprinted.

Paeth et al (1991) have patented the use of electrode 'strips' which incorporate regions of extensibility between adjacent pairs of electrodes. This, they claim, allows the selective positioning of electrodes on the body. The use of several such 'strips' should therefore enable adjacent electrodes to move relative to each other in two dimensions. Using this electrode design, it should be possible to accommodate variations in inter-electrode distance between patients as well as changes in inter-electrode distance due to patient movement.

Cherne Industries, Inc. of Minneapolis, USA market a harness for cardiac mapping that has separable sections. These sections are all removably affixed to each other by series of translayer perforations. Following application of the harness, the sections may be separated from each other, thus accommodating inter-section movement. The separable sections can also be used to accommodate inter-subject variations in the monitored body segment's dimensions and shape.

Alternatively, electrodes mounted on stretchable belts or harnesses may be used. Mills and Stein (1981) describe a method of automatically determining the correct anatomic precordial positions for electrocardiograph electrodes on the chest of patients having significantly different chest sizes. This involves stretching a 'chest-piece' containing electrodes across the patient's chest until at least two of the electrodes are aligned with their corresponding positions on the chest. The other electrodes should then, it is claimed, be on their correct position. The stretchable 'chest-piece' should also accommodate changes in chest dimensions due to breathing etc.

In the Northern Ireland Bio-Engineering Centre (NIBEC) EIT harness, the sensors and leads are printed onto a flexible substrate and the substrate is then cut out in such a way as to form highly flexible 'fingers' of printed substrate (McAdams et al 1993). The electrodes are thus free to accommodate changes in inter-electrode spacing. A narrow band of stretchable adhesive foam is used to link the electrodes and thus serves to maintain the correct initial inter-electrode spacings as well as to firmly adhere the complete system to the patient.

Belts, vests, 'cradles', 'cages' etc of spring-loaded electrode or pneumatic electrode systems should accommodate changes or variations in the body's contours or dimensions. Romberg (1988) claims the same capabilities for his inflatable electrode vest. Provided the applied pressure is not too high, such electrode systems will extend and retract along their axes. The major advantage of such electrode systems, especially the 'cradles' or 'cages' of electrodes attached to pistons, is that the positions of the electrodes could be computed and the dynamic changes in the body segment contours and the inter-electrode distances monitored. This is one of the few instances where electrodes could be free to move and yet where their relative positions would be accurately known.

\section{Conclusions}

The design of an optimal electrode system will obviously depend on the given EIT application. For short-term, resting EIr measurements, for example, it is suggested that the use of 'cradles' or 'cages' of reusable spring-Loaded electrodes or of pneumatic electrode systems appear well suited. Jossinet (1988) has pioneered the use of one such system for EIT imaging of the breast. Alternatively, in certain circumstances, suction electrode systems or inflatable vests/cuffs could be used. The low-profile, light-weight, highly flexible NIBEC EIT harness has proved successful in the long-term EIT imaging of ambulatory or bedridden patients (McAdams et al 1993) and has been used by several EIT research groups. 


\section{Acknowledgments}

The authors wish to thank N G Abraham (Charing Cross Hospital, UK), K A Grimbergen (University of Amsterdam, The Netherlands) and G Krenzke (Humboldt-Universitat zu Berlin, Germany) for kindly providing transparencies and prints of their electrode systems.

\section{References}

Abraham N G 1983 A suction electrode net for precordial ECG mapping J. Med. Eng. Tech. 7 285-7

Bergey G E, Squires R D and Sipple W C 1971 Electrocardiogram recording with pastless electrodes IEEE Trans. Biomed. Eng. BME-18 206-11

Betts R P and Brown B H 1976 Method for recording electrocardiograms with dry electrodes applied to unprepared skin Med. Biol. Eng. 14 313-15

Dempsey G J, McAdams E T, McLaughlin J A and Anderson J McC 1992 NIBEC ECG mapping harness Proc. I4th Ann. Int. Conf. IEEE Eng. Med. Biol. Soc. (Paris, 1992) (Piscataway, NJ: IEEE) pp 2702-93

Fischmann E J, Barber M R and Weiss G H 1971 Multielectrode grids which measure torso area and resistivity and yield dipole moment calibrated for these variables Vectorcardiography vol 2, ed I Hoffman, R I Hamby and E Glassman (Amsterdam: North-Holland) pp 30-41

Geddes $L$ A, Moore A G, Baker R and Mack R 1976 An easily applied and removed dry annular suction electrode J. Electrocardiol. 9 155-9

Hinsen R, Essen R V, Silny J, Merx W, Rau G and Effert S 1979 Monitoring of myocardial ischemia and necrosis in acute myocardial infarction Proc. IEEE Comput. Cardiol. 71-6

Jossinet J 1988 A hardware design for imaging the electrical impedance of the breast Clin. Phys. Physiol. Meas. 9 (Supplement A) 25-8

Kilpatrick D, Duffin P, Vickery J C and Bourdillon P J 1979 Apparatus for the rapid measurement of electrocardiographic body surface potentials Med. Biol. Eng. Comput. 17 257-60

Knorre $\mathrm{H}$, Knorre $\mathrm{M}$ and Porstmann I 1986 Elektrode fur eine Elektrodenanordnung Zur Erfassung Vielkanallger Ableitungen das Elektrokardiogramms German Patent DD 232392 A3

Kolesova G P, Chernyak V A, Gedrazhko V N and Mamchenko G F 1982 ECG suction electrodes with external vacuum pump for investigations during physical exertion Biomed. Eng. 16 31-3

Krenzke G, Dusterhoft $\tau$ and Schwanke R 1985 Vorrichtung zur Albeitung von Herzpol German Patent DD 225 $333 \mathrm{Al}$

McAdams E T, McLaughlin J, Brown B H and McArdle F 1993 The NIBEC ErT electrode harness Clinical and Physiological Applications of Electrical Impedance Tomography ed D Holder (London: UCL Press) pp 85-92

Mills H and Stein H 1981 Electrocardiograph electrodes and associated assemblies US Patent 1593594

Paeth D S, Gross S W and Lyster T D 1992 Multiple electrode strip US Patent 5191886

Romberg D 1988 Elektrodenvorrichtung zur Ableitung von Herzpotentialen German Patent DD 254523 A1

Schwesinger S, Kollande $G$ and Leipner V 1984 Electrodenguertel zur messung Bioelekrischer signale von biologischen objekten German Patent 206070

Sippensgroenewegen A, Reek E J, Spekhorst H, Broekuijsen P, Smit H W and Grimbergen K A 1986 A venturi suction electrode array for clinical body surface mapping IEEE Trans. Biomed. Eng. BME-33 $891-4$

Stein I M 1983 Electrode belt US Patent 4391279

Toyosu Y 1983 An electrocardiograph European Patent 0068032

Wen Y, Wang Z, Liu X, Zhang Y, Fang Q, Wang X and Shen C 1991 Body surface recorded cardio-electric peak analysis-diagnosis of correct position and range of myocardial infarction, prim. or sec. myocardosis and pre-excitation syndromes US Patent 5054496

Welch W 1951 Self-retaining electrocardiographic electrode J. Am. Med. Assoc. 147 1042-4

Yajima K, Kinoshita S, Tanaka $H$, Ihara $T$ and Furukawa $T 1983$ Body surface potential mapping system equipped with a microprocessor for the dynamic observation of potential patterns Med. Biol. Eng. Comput. 21 83-90 